\title{
Headache secondary to sleep-related bruxism: A case with polysomnographic findings
}

\author{
Sourav Das, Ravi Gupta, Mohan Dhyani, Deepak Goel ${ }^{1}$ \\ Departments of Psychiatry and Sleep Clinic and ${ }^{1}$ Neurology and Sleep Clinic, Himalayan Institute of Medical Sciences, Dehradun, \\ Uttarakhand, India
}

\section{ABSTRACT}

Sleep-related bruxism may present with headache. However, in clinical practice it may be difficult to differentiate from other causes of headache, especially in subjects with substance abuse. We are presenting a case of sleep-related bruxism that presented with headache and sleep-related symptoms in the presence of substance abuse. Polysomnography was used to ascertain cause of headache. How the other possible causes of headache ruled out is also discussed in report. In short, Sleep-related bruxism can cause headache that is worse in the morning. It is associated with poor quality sleep.

Key words: Headache, I sleep initiation and maintenance disorders, sleep bruxism

\section{Background}

In most of the countries, headache continues to be a major public health problem. ${ }^{[1]}$ Globally, point prevalence of headache has been estimated to be $46 \%$ and further increases to $66 \%$ when we consider the lifetime prevalence. ${ }^{[2]}$ Other than Africa, all other continents reported that nearly $50 \%$ of the general population report headache. ${ }^{[3]}$ Among all primary headaches, migraine and tension-type headaches are the most common types of primary headaches, while in the category of secondary headaches, headache attributed to head injury tops the list. ${ }^{[4]}$ Sleep and headache have an intricate and bilateral relationship. ${ }^{[5]}$ In some instances, sleep disorders may have an incremental effect on primary headaches. ${ }^{[5]}$ Further, sometimes this relationship turns into causality and a sleep disorder may give rise to a headache. ${ }^{[4]}$ Sleep-related bruxism (SRB) is one such disorder which may be associated with temporomandibular joint (TMJ)

\begin{tabular}{|l|l|}
\hline \multicolumn{2}{|c|}{ Access this article online } \\
\hline Quick Response Code: & Website: \\
\hline & www.ruralneuropractice.com \\
\cline { 2 - 3 } & \\
\hline
\end{tabular}

dysfunction and headache is often the presenting complaint. ${ }^{[6,7]}$ This kind of headache i.e. "headache attributed to TMJ disorders" has been described as a headache that has temporal relationship with the onset of pathology around TMJ; progresses or improves with the course of underlying disease; is associated with local tenderness and/or exacerbates on jaw movement. ${ }^{[4]}$

SRB is characterized by frequent or regular tooth-grinding sounds during sleep with at least one of the following: (i) Abnormal wear of the teeth; (ii) jaw muscle fatigue or pain in the morning; and/or temporal headache; and/or locked-jaw upon awakening. ${ }^{[6]}$ Bruxism can occur during wakefulness or during sleep. Awake bruxism occurs during the day in person with normal circadian sleep-wake cycle and they often develop headache during the day or in the evening. ${ }^{[6]}$ Etiologically, SRB may be either idiopathic (primary) or secondary. ${ }^{[6]}$ The secondary form is often seen in children with intellectual disability, Parkinson's disease, substances of abuse and with the use of psycho-active drugs. ${ }^{[6]}$ The prevalence of bruxism among adults is around $8 \%{ }^{[6]}$

Here we present a case of morning onset headache with poor-quality sleep and excessive daytime sleepiness that was found to be suffering from SRB. This case highlights the need for paying attention to the sleep of the individual in cases of morning onset headache.

Address for correspondence:

Dr. Ravi Gupta, Department of Psychiatry and Sleep Clinic, Himalayan Institute of Medical Sciences, Swami Ram Nagar, Doiwala, Dehradun - 248 140, Uttarakhand, India. E-mail: sleepdoc.ravi@gmail.com 


\section{Case Report}

A 40-year- old male presented with bi-temporal, mild to moderate, pressing type of headache after waking up in the morning since past 6 years, almost every day. The intensity of the headache used to decrease by the evening and was associated with teeth ache. It was non-radiating and was not associated with any of the symptoms to suggest any of the primary headaches. ${ }^{[4]} \mathrm{He}$ had a refreshing sleep and according to him, it was devoid of any abnormal activity. His usual bed time was $9 \mathrm{pm}$ and subjective sleep-onset latency was approximately 30 minutes. Habitually, he used to wake up by $5 \mathrm{am}$. He denied nocturnal awakenings and daytime napping both planned and unplanned.

However, his bed-partner's (wife's) information regarding his sleep was contradictory. According to her, he used to grind teeth almost every night during sleep. According to her, it started around 6 years back and gradually progressed in frequency. She could not cite any reason for the same. The sound of the teeth grinding was loud enough to interfere with her sleep. She had also noticed that teeth grinding used to aggravate after consumption of alcohol, smoking and during stressful periods and vice-versa.

In addition to the teeth grinding, since past 6 years, his wife also reported witnessing infrequent $(<1$ per month) episodes of abnormal activity during sleep where he would wake-up suddenly, looking fearful with eyes wide open, and sweating. He would hyperventilate, thrash his limbs and shout during these episodes. These episodes used to last few minutes and were occurring 2-3 hours after falling asleep, but never in the latter half of the night. During the episodes, he was inconsolable, confused and unresponsive to communication. He often fell asleep himself after these episodes but couldn't recall the incidence next morning. He had never injured himself or the bed-partner during these episodes. She often found him talking during the sleep, although sleep talking was decreasing in frequency over the past few years.

She had also noticed that her husband used to doze off during the day for 3 years, only during passive activities like paper reading, watching TV, during travelling and after lunch. However, this was not interfering was his day to day activities. His Epworth Sleepiness Scale (ESS) score was $12 .{ }^{[8]}$

Past history revealed that he was a smoker for 8 years and used to smoke around 20 cigarettes each day. He was also consuming $90-180 \mathrm{ml}$ of alcohol almost daily during the night for past 23 years.
There was no history to suggest teeth hypersensitivity, obstructive sleep apnea, Willies-Ekbom's disease (WED/ RLS), circadian rhythm sleep disorder, voluntary sleep restriction, psychotropic substance use, except those mentioned above and psychiatric or medical disorder in the past. The patient denied history of any of the sleep disorders during childhood. Family history was non-remarkable with reference to sleep disorders or medical disorders.

Physical examination was done according to standard protocols ${ }^{[9,10]}$ which revealed Mallampatti grade II airway, dental attrition, bilaterally tender TMJs and normal-sized muscles of mastication. Nasal airways were patent. He weighed $67 \mathrm{~kg}$; he was $169 \mathrm{~cm}$ tall and neck circumference was $36 \mathrm{~cm}$. General physical and neurological examination did not reveal any abnormality.

Clinically, a diagnosis of SRB with night terrors with multiple substance abuse with headache attributed to TMJ disorders was made and whole night video-synchronized attended polysomnography was planned.

The diagnosis was confirmed on polysomnography (see below) and he was started on a combination of tab. clonazepam $0.5 \mathrm{mg}$ hs along with tab. Ropinirole $0.25 \mathrm{mg}$ hs. He was also referred to the dentist for teeth guard. He was advised to remain abstinent from the smoking and alcohol. He came for the follow up after a month and reported partial improvement in headache, sleep quality and bruxism, although the substance abuse continued. He did not comply with the dentist's treatment.

\section{Polysomnography}

Standard 19 channel video synchronized attended diagnostic polysomnography (Cadwell Inc.) was performed according to the recent guidelines. ${ }^{[11]}$ Same guidelines were used to score the raw data. Data of the first study night was discarded as his sleep quality was not good. Here we are presenting the data of second diagnostic night. The patient did not take alcohol on the study night and sleep study was performed in the sleep laboratory of our institute.

Polysomnography findings are depicted in Table 1. Frequent rhythmic muscle movement activity (RMMA) with micro-arousals were observed during sleep with RMMA index of 11.5/hour, predominantly during N1 and N2 sleep [Figure 1]. Frequent limb movements were also observed during night with periodic limb movement during sleep (PLMS) index of 48 ]. In some epochs the limb movements came along with or preceded RMMA. Figure 1 depicts the polysomnographic picture of SRB. 
The recordings did not show evidence REM atonia or sleep talking or night terrors on either of the nights.

\section{Discussion}

This adult male presented with complaints of morning headache, non-refreshing sleep, abnormal activity during sleep and regular intake of alcohol. Polysomnography revealed RMMA during sleep with PLMS.

In this patient, the headache was not fulfilling the criteria for any of the primary headaches. ${ }^{[4]}$ However, upon clinical information, two secondary headaches-alcohol induced headache and headache attributed to TMJ disorders were considered.

Table 1: Polysomnographic variables in the case

\begin{tabular}{lc}
\hline Total sleep time & $502 \mathrm{~min}$ \\
Sleep onset latency & $6 \mathrm{~min}$ \\
Wake after sleep-onset & $21 \mathrm{~min}$ \\
Wake after sleep-offset & $0.5 \mathrm{~min}$ \\
Sleep efficiency (100xTST/SPT) & $96 \%$ \\
REM latency & $140 \mathrm{~min}$ \\
$\mathrm{~N} 1$ (\% of TST) & $8 \%$ \\
$\mathrm{~N} 2$ (\% of TST) & $62 \%$ \\
$\mathrm{~N} 3(\%$ of TST) & $5 \%$ \\
REM (\% of TST) & $21 \%$ \\
Arousal index & 18.7 \\
Respiratory disturbance index & 1 \\
Mean SpO & $95 \%$ \\
Minimum SpO & 2 \\
Snoring index & $92 \%$ \\
PLMS index & 34.4 \\
PLMS with arousal & 48.4 \\
\hline
\end{tabular}

TST: Total sleep time; SPT: Sleep period time; REM: Rapid eye movement; PLMS: Periodic limb movement during sleep
Alcohol intake can give rise to headache (alcohol induced headache) and it presents with at least one of the following characters: Bilateral; pulsating quality; and/or aggravates upon physical activity. ${ }^{[4]}$ It may start immediately, or after a few hours of alcohol consumption but both the types usually resolve within 72 hours of the last intake of alcohol..$^{[4]}$ In the present case, the headache did not meet any of the characters of alcohol-induced headache except that it was bilateral. Thus, the alcohol-induced headache could be ruled out.

However, this headache had all the characteristics of "headache attributed to TMJ disorders" (see above). ${ }^{[4]}$ He had bi-temporal headache along with pain in the teeth and tender TMJs which started after the onset of SRB. SRB was confirmed by the presence of teeth grinding, attrition of teeth, pain in teeth and bi-temporal headache especially in morning associated with non-refreshing sleep. ${ }^{[6]}$ Headache occurring during SRB often has the quality of tension-type headache and seen maximally during the morning or day. ${ }^{[6]}$ These characters were seen in this patient. One interesting fact is that severity of SRB does not correlate with the severity of symptoms. ${ }^{[6]}$

Alcohol, smoking and stress, all have been described to worsen SRB, and could explain the worsening of headache in this case. ${ }^{[12]}$

It must be noted here that the patient himself and his wife had contradictory information regarding the nocturnal events and daytime sleepiness. Moreover, the wife's information helped us in reaching to a diagnosis. Thus, in such cases information from bed-partner must be gathered.

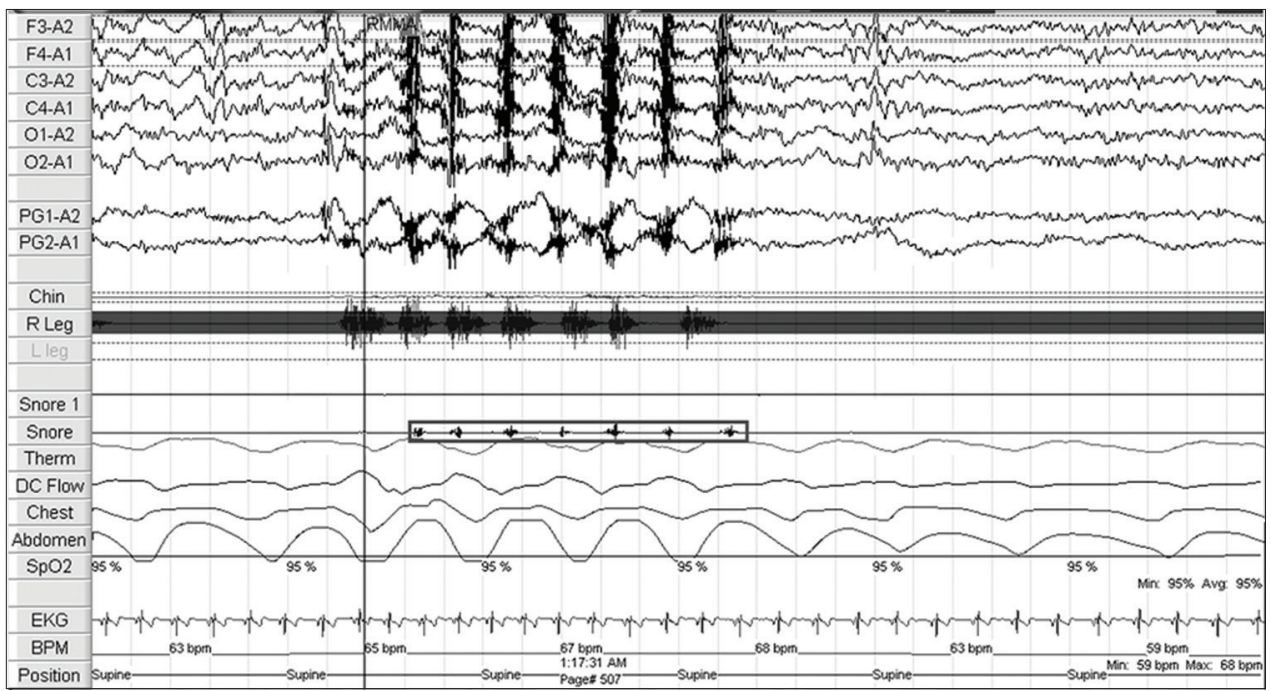

Figure 1: Polysomnography findings in sleep-related bruxism. Limb Movement (Right leg) preceding the RMMA by 1 second and associated with micro-arousal (EEG), grinding sounds (Snore), normal airflow and increase in heart rate (BPM) 
The patient also had history of occasional sleep terrors and sleep talking. These are the disorders of arousal and seen when continuity of NREM sleep is disturbed by any internal or external stimulus. ${ }^{[6]}$ SRB could be associated with partial arousals during sleep, most commonly occurring in the early part of the night. ${ }^{[6,13]}$ Thus, disorders of arousals could be seen in these patients. Further, according to one school of thought, these recurrent micro-arousals worsen the sleep quality and contribute to excessive daytime somnolence, as evident by dozing off during passive activities this case (ESS $=12$ ).

SRB often follows a micro-arousals and this was seen in this case also [Figure 1]. ${ }^{[6]}$ This subject also had periodic limb movements during sleep (PLMS) without history of Willies-Ekbom's disease, which were occasionally associated with arousals, RMMA or bruxism [Figure 1]. Earlier studies have discussed the relationship between PLMS and bruxism. PLMS index was found to be higher in patients of sleep bruxism, than the general population. ${ }^{[14]}$ Moreover, sleep bruxism and PLMS were often found together, mostly associated with EEG arousal, than any one of them in isolation. ${ }^{[14]}$ It has been hypothesized that both of these may also share the common patho-physiology. ${ }^{[14]}$ The polysomnographic findings of the this patient also corroborate these findings [Figure 1].

Differential diagnosis for SRB are sleep apnea, REM sleep behavior disorder (RBD), fascio-mandibular myoclonus, orofacial dyskinesias persisting in sleep, night-terrors, confusional arousals and rarely sleep-related epilepsy. ${ }^{[6]}$ Among these, sleep apnea was ruled out because of apnea-hypopnea index $<5$ on diagnostic study; RBD by history and absence of muscle atonia during REM sleep. He did not have any orofacial dyskinesia during wakefulness, hence their persistence during sleep was ruled out. Sleep-related epilepsy is a rare condition that can produce SRB. EEG did show any evidence of epileptic activity during any of these episodes, hence, it could be ruled out. Although it must be noted here that standard EEG montage is required to rule out sleep-related epilepsy. Fascio-mandibular myoclonus is another condition that mimics SRB and seen more commonly in patients with RBD ${ }^{[6]}$ However, in this condition, the muscle bursts are of shorter duration ( $<250 \mathrm{~ms}$ ) as compared to bursts of SRB/RMMA that are 250-2000 $\mathrm{ms}$ in duration [Figure 1]. ${ }^{[6]}$
The idea behind reporting the case is to sensitize the readers about a common yet overlooked condition in clinical practice. Patients with headache, particularly those with bi-temporal headache, pain in the jaws, and non-refreshing sleep should be evaluated for SRB. In such cases, parallel information from bed-partner must be gathered. Inspection for dental attrition, palpation of the jaw muscles and tender TMJ can be good screening tools for the same.

\section{References}

1. Stovner LJ, Zwart JA, Hagen K, Terwindt GM, Pascual J. Epidemiology of headache in Europe. Eur J Neurol 2006;13:333-45.

2. Stovner Lj, Hagen K, Jensen R, Katsarava Z, Lipton R, Scher A, et al. The global burden of headache: A documentation of headache prevalence and disability worldwide. Cephalalgia 2007;27:193-210.

3. Stovner LJ, Andree C. Prevalence of headache in Europe: A review for the Eurolight project. J Headache Pain 2010;11:289-99.

4. Headache Classification Committee of the International Headache Society (IHS). The International Classification of Headache Disorders, $3^{\text {rd }}$ ed (beta version). Cephalalgia 2013;33:629-808.

5. Gupta R, Lahan V, Goel D. Primary headaches in restless legs syndrome patients. Ann Indian Acad Neurol 2012;15 Suppl 1:S104-8.

6. American academy of sleep medicine. International Classification of Sleep Disorders. $3^{\text {rd }}$ ed. Diagnostic and coding manual. Westchester, Illinois, USA: American Academy of Sleep Medicine. 2014. p.

7. de Siqueira SR, Vilela TT, Florindo AA. Prevalence of headache and orofacial pain in adults and elders in a Brazilian community: An epidemiological study. Gerontology 2013. [Epub ahead of print].

8. Johns MW. A new method for measuring daytime sleepiness: The Epsworth sleepiness scale. Sleep 1991;14:540-5.

9. Mongini F. Headache and Facial Pain. Stuttgart: Thieme; 1999.

10. Cao MT, Guilleminault C, Kushida CA. Clinical features and evaluation of obstructive sleep apnea and upper airway resistance syndrome. In: Kyger MH, Roth T, Dement WC, editors. Principles and Practice of Sleep Medicine. Philadelphia PA: Elsevier Saunders; 2011. p. 1206-18.

11. Berry RB, Brooks R, Gamaldo CE, Harding SM, Lloyd RM, Marcus CL, et al. The AASM Manual for the Scoring of Sleep and Associated Events: Rules, Terminology and Technical Specifications. Version 2.0.1. Darien, Illinois, USA: American Academy of Sleep Medicine; 2013.

12. Cuccia AM. Etiology of sleep bruxism: A review of the literature. Recenti Prog Med 2008;99:322-8.

13. Wills L, Garcia J. Parasomnias: Epidemiology and management. CNS Drugs 2002;16:803-10.

14. van der Zaag J, Naeije M, Wicks DJ, Hamburger HL, Lobbezoo F. Time-linked concurrence of sleep bruxism, periodic limb movements, and EEG arousals in sleep bruxers and healthy controls. Clin Oral Investig 2014;18:507-13.

How to cite this article: Das S, Gupta R, Dhyani M, Goel D. Headache secondary to sleep-related bruxism: A case with polysomnographic findings. J Neurosci Rural Pract 2015;6:248-51.

Source of Support: Nil. Conflict of Interest: None declared. 